\title{
The Tautenburg Schmidt Telescope and Galactic Proper Motion Studies
}

\author{
E. Schilbach, R.-D. Scholz, S. Hirte \\ Universität Potsdam/Sternwarte Babelsberg, An der Sternwarte 16, \\ 14482 Potsdam, Germany
}

\begin{abstract}
The combination of Tautenburg plates and automatic measuring machines provides a powerful tool to obtain photometry and proper motions of a great number of stars for statistical investigations of our Galaxy. Photographic photometry with an accuracy of about $0.07 \mathrm{mag}$ can be obtained provided two plates of the same colour and a sufficient number of photometric standards are available. With two plate pairs and a 20 years baseline, a proper motion accuracy better than 4 mas/year can be achieved for stars over a wide range of magnitudes. Outside the Galactic plane proper motions are determined with respect to hundreds of background galaxies.
\end{abstract}

\section{Introduction}

Deep Schmidt plates covering large sky areas provide an enormous amount of information required for detailed stellar statistical studies of the Galaxy. Since automated measuring machines have become available it is no longer a problem to extract this information from a Schmidt plate. Nevertheless, to obtain reliable results we have to take into account possible local systematic effects which could arise as a consequence of the wide field, the necessary bending of Schmidt plates during exposure etc. According to the general view taken now, for the global Schmidt plate solution one has to use high order polynomials. The polynomial order is chosen in dependence on instrumental parameters of the telescope and on the size of the investigated field.The accuracy of the polynomial coefficients results not only from data precision but also from the number of reference points included in the astrometric reduction.

For a differential proper motion determination thousands of reference stars can be selected among the faint stars with small proper motions. Outside the Galactic plane, the link to an extragalactic reference system can then be carried out with respect to hundreds of galaxies. At high Galactic latitudes with even thousands of galaxies a global plate-to-plate solution is possible by direct use of the galaxies as reference points. On the other hand, a global plate-to-catalogue solution does not yield high positional accuracy over the whole field because of an insufficient density of precise catalogue stars. A significant improvement of the astrometric reduction of Palomar Schmidt plates has been achieved by non-conventional methods proposed by Taff et al. (1990), Lattanzi et al. (1991) and Röser (this Vol.). Nevertheless, the low number of reference stars remains a weak point in the determination of positions from Schmidt plate measurements. 
With the Tautenburg Schmidt telescope (134/200/400) more than 8000 plates have been taken in selected Northern sky areas since it was mounted in 1960. A $24 \mathrm{~cm} \times 24 \mathrm{~cm}$ Tautenburg plate covers a field of about 10 square degrees, and a 20 minute exposure of a $B$ plate has a limiting magnitude of 19 to 21. In comparison to other large Schmidt telescopes, irregular local shifts of the emulsion caused by the rebending after the exposure are of less influence due to the four meter focal length and the use of relatively small plates. The large focal length leads to a plate scale of $51 \mathrm{arcsec} / \mathrm{mm}$ providing a relatively high positional accuracy. Regarding the instrumental parameters, most promising tasks for astrometric use of the Tautenburg telescope are the determination both of stellar proper motions in selected areas and positions of extragalactic radio sources (ERS).

\section{Astrometry}

Errors of mean stellar positions in dependence on B magnitude obtained from plate-to-plate solutions of Tautenburg plates measured with the MAMA machine in Paris are given in Table 1. Except for stars brighter than $B=11$, a similar accuracy is achieved for Tautenburg plates measured with the APM facility in Cambridge (Schilbach \& Scholz 1992). For both machines, a positional accuracy of less than $1 \mu \mathrm{m}$ is typical over a wide range of magnitudes and becomes much worse at about one magnitude before the plate limit.

To test the distribution of the internal proper motion accuracy over the field, the proper motions of 8490 stars obtained from MAMA measurements of four overlapping plate pairs with a 29 years baseline were used. About 4000 anonymous stars in an intermediate range of magnitudes were selected as reference. Dividing the measured field into 10 by 10 sub-areas we averaged the rms of proper motion in each sub-area. The mean accuracy of proper motions varied from 1 to 3 mas/year with no systematic effects over the field.

The plate reduction is carried out with a complete third-order polynomial. Higher order terms in the Tautenburg plate-to-plate solution do not improve the accuracy of final results.

\section{Photometry}

APM measurements of Tautenburg plates are internally calibrated so that the photometric zero-point can be defined with only a few photoelectric standards. For converting the MAMA fluxes to magnitudes a sufficient number of standards covering the whole range of magnitudes is necessary due to the nonlinear relationship.

According to the technical concept confirmed by numerous tests, the Tautenburg telescope is vignetting-free over the whole field covered by a Tautenburg plate. Small systematic effects depending on the plate positions have been found (Andruk et al. 1994) though their values vary from plate to plate. This fact may be better explained by different conditions during exposure (e.g. local atmospheric effects), developing and measuring or by systematic changes of emulsion properties over the plates than by aberrations related to the telescope and filter alone. As proved by several investigations the Johnson UBV system is well realized by the Tautenburg telescope. 
The internal accuracy of $B$ and $V$ magnitudes obtained from MAMA measurements of Tautenburg plates is given in Table 1.

Table 1. Internal accuracy of positions and photographic magnitudes from measurements of two Tautenburg plates .

\begin{tabular}{ccccc}
$\begin{array}{c}\text { magnitude } \\
\text { interval }\end{array}$ & $\begin{array}{c}\text { rms }(\mathrm{X}) \\
(\mu \mathrm{m})\end{array}$ & $\begin{array}{c}\text { rms }(\mathrm{Y}) \\
(\mu \mathrm{m})\end{array}$ & $\begin{array}{c}\text { B band } \\
(\mathrm{mag})\end{array}$ & $\begin{array}{c}\mathrm{V} \text { band } \\
(\mathrm{mag})\end{array}$ \\
\hline $10 \leq 10$ & 1.0 & 0.9 & 0.05 & 0.05 \\
$10 \leq m<11$ & 0.8 & 1.1 & 0.05 & 0.05 \\
$11 \leq m<12$ & 0.6 & 0.7 & 0.05 & 0.05 \\
$12 \leq m<13$ & 0.6 & 0.6 & 0.05 & 0.06 \\
$13 \leq m<14$ & 0.6 & 0.6 & 0.05 & 0.06 \\
$14 \leq m<15$ & 0.6 & 0.8 & 0.05 & 0.06 \\
$15 \leq m<16$ & 0.7 & 0.6 & 0.04 & 0.06 \\
$16 \leq m<17$ & 0.9 & 0.9 & 0.04 & 0.07 \\
$17 \leq m<18$ & 1.4 & 1.3 & 0.05 & 0.09 \\
$18 \leq m<19$ & 2.4 & 2.3 & 0.08 & 0.09 \\
$19 \leq m$ & 4.5 & 4.3 & 0.10 & \\
\hline \hline
\end{tabular}

\section{Programmes}

According to Table 1, a proper motion accuracy better than 4 mas/year (with two Tautenburg plate pairs and a 20 years baseline) and a photometric accuracy of about $0.05 \mathrm{mag}$ (from two plates of the same colour) can be achieved for stars with $B<18$.

About 3000 photographic plates were taken in Tautenburg before 1970. As the Tautenburg Schmidt was not designed for a sky survey but predominantly used for a detailed study of selected areas there are a few fields with more than 100 plates and 70 fields with at least 4 old plates. These observations present an important potential for different proper motion programmes.

Link of the HIPPARCOS catalogue to an extragalactic reference frame Within the activities of the Working group "HIPPARCOS Link to the Extragalactic Reference Frame" we determine absolute proper motions of HIPPARCOS stars directly with respect to galaxies and optical positions of ERS using Tautenburg plates. With absolute proper motions of about 600 link stars in ca. 50 fields well distributed over the Northern sky, we expect to reduce the residual rotation of the HIPPARCOS system to less than 1 mas/year. This provides nearly the same accuracy as VLBI observations.

The Potsdam programme to determine optical positions of ERS includes 42 objects for which at least two Tautenburg Schmidt plates centered on the ERS had been taken from 1986 to 1992 . For 11 sources the external positional accuracy in $(\alpha \cos \delta, \delta)$ has been estimated from comparison with radio positions by Dick et al. (1991) as $(0.20,0.29)$ arcsec with the AGK3 and $(0.13,0.18)$ arcsec with the PPM as reference catalogue. HIPPARCOS will provide positions of about 25 stars per 10 square degrees with an accuracy of less than 2 mas so that in any Tautenburg plate solution only the errors of the Schmidt plate and of the measuring process will appear. We checked the ability to get accurate positions in the centre of Tautenburg plates by a comparison of MAMA measurements of 
5 plate pairs close to the HIPPARCOS epoch with the HIPPARCOS 18 months solution. The mean positional accuracy of a HIPPARCOS star near the plate centre which has not been included in the solution is $(0.08,0.10)$.

Kinematics of stars in the main Galactic meridian

In the framework of the programme of studying the meridional section of the Galaxy (MEGA) in 17 fields, the absolute proper motions of about 9000 stars with respect to 3000 galaxies and their $B, V$ magnitudes have been determined in two sky regions near the north Galactic Pole (NGP) (Kharchenko et al. 1994). The completeness $B$ magnitude is $18.3 \mathrm{mag}$ and the investigated field covers 14.6 square degrees of the sky. Distances have been determined using the stellar magnitudes, colours, proper motions and reduced proper motions. Stellar kinematics, eccentricities of Galactic orbits, spatial distribution and changes of these characteristics with $Z$-distance from the Galactic plane are obtained up to $15 \mathrm{kpc}$. Four subsystems have been found and described in the NGP direction. Kinematical interpretation of data for ca. 100,000 stars in the direction of the Galactic centre and anticentre is in progress.

Kinematics of star clusters

Proper motions for ca. 40,000 stars in the Pleiades have been determined by use of 12 partly overlapping pairs of Tautenburg plates. An accuracy of 2 mas/year has been achieved for the majority of stars up to 18th mag. The data are used for the determination of the Pleiades membership and a kinematical study within a ca. 17 square degree region centred near Alcyone (Souchay et al., this Vol.).

Mean absolute proper motions of the globular clusters M3 and M92 were derived with an accuracy of 0.6 mas/year from 5 pairs of Tautenburg plates. The data have been used for the determination of galactic orbits of the clusters (see Scholz et al. 1993). Further APM measurements of Palomar and Tautenburg Schmidt plates with seven other globulars have been completed.

\section{Acknowledgments}

We thank the Tautenburg staff for supplying the plates and the MAMA and APM teams for measuring the plates.

\section{References}

Andruk V., Kharchenko N. \& Schilbach E., 1994, Astron.Nachr., 315, in press Dick W. R. \& Kumkova I. I., 1991, in Problems of Astrometry and Space Geodynamics, N. V. Kharchenko, ed., (Naukova Dumka, Kiev) p. 51

Lattanzi M. G. \& Bucciarelli B., 1991, A\&A, 250, 565

Kharchenko N., Schilbach E. \& Scholz R.-D., 1994, Astron.Nachr., 315, in press

Röser S., 1994, these proceedings

Schilbach E. \& Scholz R.-D., 1992, Astron. Nachr., 313, 243

Scholz R.-D., Odenkirchen M. \& Irwin M. J., 1993, MNRAS, 264, 579

Souchay J. \& Schilbach E., 1994, these proceedings

Taff L. G., Lattanzi M. G. \& Bucciarelli B., 1990, ApJ, 358, 359 